\title{
ON LINEAR ALGEBRAIC SEMIGROUPS
}

BY

MOHAN S. PUTCHA

\begin{abstract}
ABSTRACr. Let $K$ be an algebraically closed field. By an algebraic semigroup we mean a Zariski closed subset of $K^{n}$ along with a polynomially defined associative operation. Let $S$ be an algebraic semigroup. We show that $S$ has ideals $I_{0}, \ldots, I_{t}$ such that $S=I_{t} \supseteq \cdots \supseteq I_{0}, I_{0}$ is the completely simple kernel of $S$ and each Rees factor semigroup $I_{k} / I_{k-1}$ is either nil or completely 0 -simple $(k=1, \ldots, t)$. We say that $S$ is connected if the underlying set is irreducible. We prove the following theorems (among others) for a connected algebraic semigroup $S$ with idempotent set $E(S)$. (1) If $E(S)$ is a subsemigroup, then $S$ is a semilattice of nil extensions of rectangular groups. (2) If all the subgroups of $S$ are abelian and if for all $a \in S$, there exists $e \in E(S)$ such that $e a=a e=a$, then $S$ is a semilattice of nil extensions of completely simple semigroups. (3) If all subgroups of $S$ are abelian and if $S$ is regular, then $S$ is a subdirect product of completely simple and completely 0-simple semigroups. (4) $S$ has only trivial subgroups if and only if $S$ is a nil extension of a rectangular band.
\end{abstract}

1. Preliminaries. Throughout this paper, $\mathrm{Z}^{+}$will denote the set of all positive integers. If $X$ is a set, then $|X|$ denotes the cardinality of $X . K$ will denote a fixed algebraically closed field. If $n \in \mathbf{Z}^{+}$, then $K^{n}=K \times \cdots \times K$ is the affine $n$ space and $\mathfrak{N}_{n}(K)$ the algebra of all $n \times n$ matrices. If $A \in \mathfrak{K}_{n}(K)$, then $\rho(A)$ is the rank of $A$. In this paper we only consider closed sets with respect to the Zariski topology. So $X \subseteq K^{n}$ is closed if and only if it is the set of zeroes of a finite set of polynomials on $K^{n}$. Let $X \subseteq K^{m}, Y \subseteq K^{n}$ be closed, $\varphi: X \rightarrow Y$. If $\varphi=$ $\left(\varphi_{1}, \ldots, \varphi_{m}\right)$ where each $\varphi_{i}$ is a polynomial, then $\varphi$ is a morphism. Let $p, n \in \mathbf{Z}^{+}$, $p<n$. Then we use, without further comment, the well-known fact that the set $T=\left\{A \mid A \in \mathfrak{T}_{n}(K), \rho(A)<p\right\}$ is closed. In fact for $A \in \mathfrak{T}_{n}(K), A \in T$ if and only if all minors of $A$ of order $\geqslant p$ vanish. By an algebraic semigroup we mean $(S, \circ)$ where $\circ$ is an associative operation on $S, S$ is a closed subset of $K^{n}$ for some $n \in Z^{+}$and the map $(x, y) \rightarrow x \circ y$ is a morphism from $S \times S$ into $S$. If $S$ has an identity element then $S$ is an algebraic monoid. Polynomially defined associative operations on a field have been studied by Yoshida [21], [22], Plemmons and Yoshida [13]. Yoshida's results have been generalized to integral domains by Petrich [12]. Clark [4] has studied semigroups of matrices forming a linear variety. Algebraic monoids are briefly encountered in Demazure and Gabriel [8]. The author [15] has studied semigroups on affine spaces defined by polynomials of degree at most 2 .

Let $S$ be an arbitrary semigroup. If $S$ has an identity element, then $S^{1}=S$. Otherwise $S^{1}=S \cup\{1\}, 1 \notin S$, with obvious multiplication. If $a \in S$, then the

Received by the editors November 14, 1978 and, in revised form, August 29, 1979.

1980 Mathematics Subject Classification. Primary 20M10; Secondary 20 G99.

Key words and phrases. Linear algebraic semigroup, idempotent, subgroup, $g$-class.

(c) 1980 American Mathematical Society 0002-9947/80/0000-0257/\$04.25 
centralizer of $a$ in $S, C_{S}(a)=\{x \mid x \in S, x a=a x\}$. Then center of $S, C(S)=$ $\cap{ }_{a \in S} C_{S}(a)$. If $a, b \in S$, then $a \mid b$ ( $a$ divides $b$ ) if $b \in S^{1} a S^{1} \cdot \mathcal{G}, \mathcal{R}, \mathcal{L}, \mathcal{H}$ will denote the usual Green's relations on $S$ (see [6]). If $a \in S$, then we let $J(a)=$ $S^{1} a S^{1} . J_{a}, H_{a}$ will denote the $\mathcal{G}$-class and $\mathcal{H}$-class of $a$ in $S$, respectively. $E(S)$ will denote the set of idempotents of $S$. If $e, f \in E(S)$ then $e<f$ if $e f=f e=e$. An idempotent semigroup is called a band. A commutative band is called a semilattice. A band satisfying the identity $x y z w=x z y w$ is called a normal band. If $a b=b[b a$ $=b$ ] for all $a, b \in S$, then $S$ is a right [left] zero semigroup. A direct product of a right zero semigroup and a left zero semigroup is a rectangular band. A direct product of a rectangular band and a group is a rectangular group. A direct product of right [left] zero semigroup and a group is a right [left] group. Let $I$ be an ideal of $S$. If $S / I$ is a nil semigroup, then we say that $S$ is a nil extension of $I$. Let $\delta_{\alpha}$ ( $\alpha \in \Gamma$ ) be a set of congruences on $S$. If $\cap_{\alpha \in \Gamma} \delta_{\alpha}$ is the equality congruence, then $S$ is a subdirect product of $S_{\alpha}(\alpha \in \Gamma)$. See [7, p. 99] for details.

A congruence $\delta$ on $S$ is an $\mathcal{\delta}$-congruence if $S / \delta$ is a semilattice. If $S$ is a disjoint union of subsemigroups $S_{\alpha}(\alpha \in \Gamma)$ and if for each $\alpha, \beta \in \Gamma$, there exists $\gamma \in \Gamma$ such that $S_{\alpha} S_{\beta} \cup S_{\beta} S_{\alpha} \subseteq S_{\gamma}$, we will say that $S$ is a semilattice (union) of $S_{\alpha}$ $(\alpha \in \Gamma)$. We also say that $S_{\alpha}(\alpha \in \Gamma)$ is a semilattice decomposition of $S$. There is an obvious natural correspondence between $\mathcal{S}$-congruences and semilattice decompositions [6, p. 25]. A semigroup with no $\delta$-congruences other than $S \times S$ is said to be $\mathcal{S}$-indecomposable. Let $\xi$ be the finest $\mathcal{S}$-congruence on $S$. Throughout this paper, we let $\Omega=\Omega(S)=S / \xi$ denote the maximal semilattice image of $S$. By a theorem of Tamura [18], [19], each component of $\xi$ is $\mathcal{S}$-indecomposable and is called the $\delta$-indecomposable component of $S$. An ideal $P$ of $S$ is prime if $S \backslash P$ is a subsemigroup of $S$. In such a case $\{P, S \backslash P\}$ is a semilattice decomposition of $S$. If $S$ is a commutative algebraic semigroup, then it follows from Corollary 1.4 below, and Tamura and Kimura [20] that $E(S)=\Omega(S)$.

Let $S, T$ be algebraic semigroups, $\varphi: S \rightarrow T$ a (semigroup) homomorphism. Then $\varphi$ is a *-homomorphism if $\varphi$ is also a morphism (of varieties). If $\varphi$ is a bijection and if both $\varphi$ and $\varphi^{-1}$ are *-homomorphisms, then we say that $\varphi$ is a ${ }^{*}$-isomorphism and that $S, T$ are *-isomorphic. $S$ is connected if the underlying closed set is irreducible (i.e. is not a union of two proper closed subsets).

The proof of the following result can be found in Demazure and Gabriel [8, II, §2, Theorem 3.3].

THeOREM 1.1 (SEE [8]). Let $S$ be an algebraic monoid. Then $S$ is *-isomorphic to a closed submonoid of $\mathfrak{T}_{n}(K)$ for some $n \in \mathbf{Z}^{+}$.

Let $S$ be an algebraic semigroup which is a group. Let $\varphi: S \rightarrow \Re_{n}(K)$ be given by Theorem 1.1. By Hilbert's Nullstellensatz, $1 / \operatorname{det} \varphi(x)$ is a polynomial on $S$. So $x^{-1}=\varphi^{-1}(\operatorname{adj} \varphi(x) / \operatorname{det} \varphi(x))$. Hence the map $x \rightarrow x^{-1}$ is a morphism and $S$ is an algebraic group in the usual sense [2].

The following result can also be found in [8, II, §2, Corollary 3.6].

COROLlARY 1.2 (SEE [8]). Let $S$ be an algebraic monoid which is not a group. Then the nonunits of $S$ form a closed prime ideal of $S$. 
Let $S$ be an algebraic semigroup, $S \subseteq K^{n}$. Let $T=(S \times\{0\}) \cup\{(0,1)\} \subseteq$ $K^{n+1}$. Define $(x, \alpha)(y, \beta)=(x y+\beta x+\alpha y, \alpha \beta)$. Then $T$ is an algebraic monoid with identity element $(0,1) . S$ is *-isomorphic to the closed subsemigroup $S \times\{0\}$ of $T$. Hence we have

COROllary 1.3. Let $S$ be an algebraic semigroup. Then $S$ is *-isomorphic to a closed subsemigroup $\mathfrak{T}_{n}(K)$ for some $n \in \mathbf{Z}^{+}$.

The following result was pointed out to the author by Clark [5].

COROllary 1.4 [Clark]. Let $S$ be an algebraic semigroup. Then there exists $n \in \mathbf{Z}^{+}$such that for all $a \in S, a^{n}$ lies in a subgroup of $S$.

Proof. By Corollary 1.3, we can assume that $S$ is a closed subsemigroup of $\mathfrak{T}_{n}(K)$ for some $n \in \mathrm{Z}^{+}$. Let $A \in S$. Then without loss of generality we can assume $A=\left(\begin{array}{c}B: 0 \\ 0\end{array}: \dot{C}\right)$. where $B \in \mathfrak{N}_{p}(K)$ is invertible and $C \in M_{n-p}(K)$ is nilpotent. Let $T=\left\{\left(\begin{array}{c}X: 0 \\ 0: 0\end{array}\right) \mid X \in M_{p}(K), X\right.$ is invertible $\}$. Then $A^{n} \in T$. Let $G=\left\{\left(\left(\begin{array}{l}X: 0 \\ 0: 0\end{array}\right), \alpha\right) \mid X\right.$ $\left.\in M_{p}(K), \alpha \in K, \alpha \operatorname{det} \cdot X=1\right\}$. Then $G$ is an algebraic group, $\varphi: G \rightarrow T$ given by $\varphi\left(\left(\begin{array}{l}X: 0 \\ 0: 0\end{array}\right), \alpha\right)=X$ is a bijective morphism. $G_{1}=\varphi^{-1}(T \cap S)$ is a closed subsemigroup of $G_{1}$. It is well known (see [8, II, §2, Corollary 3.5]) that a closed submonoid (and hence a closed subsemigroup) of a linear algebraic group is a subgroup. Thus $G_{1}$ is a subgroup of $G$. So $T \cap S=\varphi\left(G_{1}\right)$ is a subgroup of $S$. Since $A^{n} \in T \cap S$, we are done.

COROllaRY 1.5. Let $S$ be an algebraic semigroup. Then $S$ has a kernel $M$ which is closed and completely simple.

Proof. By Theorem 1.1, we can assume that $S$ is a closed subsemigroup of $\mathfrak{T}_{n}(K)$ for some $n \in \mathbf{Z}^{+}$. By Corollary 1.4 and Clark [3], $S$ has a completely simple kernel $M$ given by its elements of minimal rank $r$. Then $M=\{a \mid a \in S$, $\rho(a)<r+1\}$ is closed.

LEMMA 1.6. Let $\mathcal{E}$ be an infinite set of idempotents in $\mathfrak{T}_{n}(K)$ of rank $r$. Then there exist $E, F \in \mathcal{E}$ such that $E \neq F$ and $\rho(E F)=\rho(F E)=r$.

Proof. If $E \in \mathcal{E}$, then let $\mathbb{Q}_{E}=\left\{A \mid A \in \mathfrak{T}_{n}(K), \rho(E A)<r\right\}, \mathscr{B}_{E}=\{A \mid A \in$ $\left.\mathfrak{T}_{n}(K), \rho(A E)<r\right\}$. Then $\mathbb{Q}_{E}, \mathscr{G}_{E}$ are closed subsets of $\mathfrak{T}_{n}(K)$. We claim:

There exists an infinite subset $\mathscr{F}$ of $\mathcal{E}$ such that for all $E \in \mathscr{F},\left|\mathbb{Q}_{E} \cap \mathcal{F}\right|<\infty$.

Suppose (1) is false. Then there exists $E_{1} \in \mathcal{E}$ such that $\mathcal{E}_{1}=\mathbb{Q}_{E_{1}} \cap \mathcal{E}$ is infinite. Again by (1), there exists $E_{2} \in \mathcal{E}_{1}$ such that $\mathcal{E}_{2}=\mathscr{Q}_{E_{2}} \cap \mathcal{E}_{1}$ is infinite. Continuing, we obtain a sequence $E_{1}, E_{2}, \ldots$, in $\delta$ such that $\rho\left(E_{i} E_{j}\right)<r$ for $i<j$. So $E_{i+1} \in \mathbb{Q}_{E_{1}} \cap \cdots \cap \mathbb{Q}_{E_{i}}, E_{i+1} \notin \mathbb{Q}_{E_{i+1}}$. Hence

$$
\mathbb{Q}_{E_{1}} \supsetneqq \mathbb{Q}_{E_{1}} \cap \mathbb{Q}_{E_{2}} \supsetneqq \mathbb{Q}_{E_{1}} \cap \mathbb{Q}_{E_{2}} \cap \mathbb{Q}_{E_{2}} \supsetneqq \cdots
$$

Since $\mathbb{Q}_{E_{i}}$ 's are closed sets, we have a contradiction to the Hilbert Basis Theorem. Thus (1) is true. The dual of (1) applied to $\mathscr{F}$ shows that there exists an infinite subset $\mathcal{G}$ of $\mathscr{F}$ such that for all $E \in \mathcal{G},\left|\mathscr{B}_{E} \cap \mathcal{G}\right|<\infty$. Let $E \in \mathcal{G}$. Then 
$\left|\mathbb{Q}_{E} \cap \mathcal{G}\right|<\infty,\left|\mathscr{B}_{E} \cap \mathcal{G}\right|<\infty$. Hence there exists $F \in \mathcal{G}$ such that $F \notin \mathbb{Q}_{E} \cup$ $\mathscr{B}_{E}$. So $\rho(E F)=\rho(F E)=r$.

A semigroup $S$ with the property that a power of each element lies in a subgroup of $S$ is said to be strongly $\pi$-regular. The study of strongly $\pi$-regular rings and semigroups was initiated by Azumaya [1], Drazin [9] and Munn [11]. Clark [3] showed that a strongly $\pi$-regular matrix semigroup has a kernel given by its elements of minimal rank. Let $S$ be a strongly $\pi$-regular semigroup. A $g$-class of $S$ containing an idempotent is called regular.

THEOREM 1.7. Let $S$ be a strongly $\pi$-regular subsemigroup of $\mathfrak{R}_{n}(K)$. Then $S$ has only finitely many regular $q$-classes.

Proof. Suppose not. Then there exists an infinite set of idempotents $\mathcal{E}$ of $S$ such that for all $e, f \in \mathcal{E}, e \mathscr{f} f$ implies $e=f$. Let $r=0, \ldots, n$, let $\mathcal{E}_{r}=\{e \mid e \in \mathcal{E}$, $\rho(e)=r$. Then $\mathcal{E}_{r}$ is infinite for some $r$. By Lemma 1.6, there exist $e, f \in \mathcal{E}_{r}$ such that $e \neq f, \rho(e f)=\rho(f e)=r$. Let $\mathcal{V}$ be the space of all $n \times 1$ vectors on $K$. Then $e f \mathfrak{V}=e^{\mathscr{V}}, f e^{\mathscr{V}}=f^{\mathfrak{V}}$. Hence $e \mathfrak{V}=(e f)^{t} \mathfrak{V}$ for all $t \in \mathbf{Z}^{+}$. There exists an idempotent $g$ of $S, p \in \mathbf{Z}^{+}$such that $g \mathcal{H}(e f)^{p}$. Hence $g \mathcal{V}=(e f)^{p} \mathcal{V}=e \mathfrak{V}$. If $v \in \mathcal{V}$, then $e v \in g \mathcal{V}$ and so $g e v=e v$. So $g e=e$. Hence $f\left|(e f)^{p}\right| g \mid e$. So $f \mid e$. Similarly $e \mid f$ and $e g f$. This contradiction proves the theorem.

COROllary 1.8. Let $S$ be a strongly $\pi$-regular subsemigroup of $\mathfrak{T}_{n}(K)$. Then $\Omega(S)$ is finite.

Proof. Let $\varphi: S \rightarrow \Omega(S)$ denote the natural homomorphism. By Theorem 1.7, $\varphi(E(S))$ is finite. Let $a \in S$. Then $a^{n} \mathcal{H}$ Ce for some $e \in E(S)$. So $\varphi(a)=\varphi\left(a^{n}\right)=$ $\varphi(e)$. Hence $\Omega(S)=\varphi(S)=\varphi(E(S))$ is finite.

LEMMA 1.9. Let $S$ be a strongly $\pi$-regular semigroup with only finitely many regular $g$-classes. Then there exist finitely many ideals $I_{0}, \ldots, I_{t}$ of $S$ such that $S=I_{t}$ $\supseteq \cdots \supseteq I_{0}, I_{0}$ is the completely simple kernel of $S$ and each $I_{i} / I_{i-1}$ is either completely 0 -simple or a nil semigroup $(i=1, \ldots, t)$.

Proof. We prove by induction on the number of regular $g$-classes of $S$. Let $E=E(S)$. Let $J_{e_{1}}, \ldots, J_{e_{n}}$ be the regular $g$-classes of $S$ where $e_{1}, \ldots, e_{n} \in E$. Let $I=J\left(e_{1}\right) \cap \cdots \cap J\left(e_{n}\right)$. Then $I$ is an ideal of $S$. So there exists $f \in I \cap E$. Let $a \in S$. Then there exists $m \in \mathbf{Z}^{+}$such that $a^{m} g e_{i}$ for some $i$. So $f \in J\left(a^{m}\right) \subseteq J(a)$. Hence $J(f)=I_{0}$ is the kernel of $S$. By Munn [11], $I_{0}$ is completely simple. Let $\mathscr{K}=\left\{J(e) \mid e \in E \cap\left(S \backslash I_{0}\right)\right\}$. Then $\mathscr{K}$ is finite. If $\mathscr{K}=\varnothing$, then $S \backslash I_{0}$ has no idempotent and $S \backslash I_{0}$ is nil. So assume $\mathscr{K} \neq \varnothing$. Then $\mathscr{K}$ has a minimal element $J(g), g \in E$. Let $I_{2}=J(g), I_{1}=I_{2} \backslash J_{g}$. Then $I_{0} \subseteq I_{1}$ and $I_{1}$ is an ideal of $S$. Let $a \in I_{1}$. Then $a^{m} g h$ for some $h \in E, m \in \mathbf{Z}^{+}$. Then $h \in I_{1}$. So $J(h) \varsubsetneqq J(g)$. By minimality of $J(g), h \in I_{0}$. Thus $a^{m} \in I_{0}$. So $I_{1} / I_{0}$ is nil. Since $I_{2} \backslash I_{1}=J_{g}$ and $g \in E, I_{2} / I_{1}$ is 0 -simple. By Munn [11], $I_{2} / I_{1}$ is completely 0 -simple. Clearly $S / I_{2}$ has lesser number of regular $G$-classes than $S$. We are thus done by our induction hypothesis.

By Theorem 1.7 and Lemma 1.9 we have the following. 
THEOREM 1.10. Let $S$ be a strongly $\pi$-regular subsemigroup of $\Re_{n}(K)$. Then there exist ideals $I_{0}, \ldots, I_{t}$ of $S$ such that $S=I_{t} \supseteq \cdots \supseteq I_{0}, I_{0}$ is the completely simple kernel of $S$ and each $I_{i} / I_{i-1}$ is either completely 0 -simple or nil $(i=1, \ldots, t)$.

By Corollary 1.3, Corollary 1.4 and Theorem 1.10, we have

Corollary 1.11. Let $S$ be an algebraic semigroup. Then $S$ has ideals $I_{0}, \ldots, I_{t}$ such that $S=I_{t} \supseteq \cdots \supseteq I_{0}, I_{0}$ is the completely simple kernel of $S$ and each $I_{i} / I_{i-1}$ is either completely 0 -simple or nil $(i=1, \ldots, t)$.

THEOREM 1.12. Let $S$ be an algebraic semigroup and $P$ a prime ideal of $S$. Then $P$ is closed.

Proof. By Corollaries 1.3 and 1.4, we can assume that $S$ is a closed, strongly $\pi$-regular subsemigroup of $\mathfrak{T}_{n}(K)$ for some $n \in \mathbf{Z}^{+}$. Hence $S_{1}=S \backslash P$ is strongly $\pi$-regular. By Clark [3] the kernel $T$ of $S_{1}$ is the set of elements of $S_{1}$ of minimal rank. Let $e \in E(T), \rho(e)=r$. Let $a \in S_{1}$. Then $(e a e)^{n} \in T$ and so $\rho\left((e a e)^{n}\right)=r$. Let $a \in P$. There exists $f \in E(P)$ such that $(e a e)^{n} \mathcal{H} f$. So $e f=f e=f$. Hence $\rho(f)<\rho(e)=r$. Clearly $\rho\left((e a e)^{n}\right)=\rho(f)$. Thus $P=\left\{a \mid a \in S, \rho\left((e a e)^{n}\right)<r\right\}$ is closed.

2. Connected algebraic semigroups. Let $S$ be an algebraic semigroup, $e \in E(S)$. Then the maximal subgroup $H_{e}$ of $S$ need not be closed. However $H_{e}$ can be identified with $G=\{(a, b) \mid a, b \in S, a b=b a=e, a e=e a=a, b e=e b=b\}$. If $(a, b),(c, d) \in G$, define $(a, b)(c, d)=(a c, d b)$. Then $G$ is an algebraic group. The correspondence between $H_{e}$ and $G$ is given by $a \leftrightarrow\left(a, a^{-1}\right)$. More precisely define $\varphi: G \rightarrow S$ as $\varphi(a, b)=a$. Then $\varphi$ is an injective *-homomorphism and $\varphi(G)=H_{e}$. It is easy to show that $G$ is unique to within *-isomorphisms. It can also be easily shown that if $S$ is connected then so is $G$. However, we will not need these facts in this paper.

THEOREM 2.1. Let $S$ be a connected algebraic semigroup. Then $\Omega(S)$ has an identity element.

Proof. Let $\Omega=\Omega(S), \varphi: S \rightarrow \Omega$ be the canonical homomorphism. By Corollary $1.8, \Omega$ is a finite semilattice. Suppose $\Omega$ has two maximal elements $e, f$. Then $\Omega_{1}=\Omega \backslash\{e\}, \Omega_{2}=\Omega \backslash\{f\}$ are prime ideals of $\Omega, \Omega=\Omega_{1} \cup \Omega_{2}$. So $S=P_{1} \cup P_{2}$ where $P_{i}=\varphi^{-1}\left(\Omega_{i}\right), i=1,2$. But $P_{1}, P_{2}$ are prime ideals of $S$ and hence closed by Theorem 1.12. This contradiction shows that $\Omega$ has a maximum element $e$. So $e$ is the identity element of $\Omega$.

In the above notation, we call $\varphi^{-1}(e)$ the top $\delta$-indecomposable component of $S$. If $S$ is a monoid, then the top $\mathcal{S}$-indecomposable component of $S$ is the group of units of $S$.

Proposition 2.2 Let $S$ be a connected algebraic semigroup, $e, f \in E(S)$. Then $e S$, Se, eSf are connected, closed subsemigroups of $S$. If SeS is closed, then SeS is also connected. 
Proof. $e S=\{x \mid x \in S, e x=x\}, e S f=\{x \mid x \in S, e x=x=x f\}$. Hence $e S$, Se, $e S f$ are closed. Define $\varphi_{1}: S \rightarrow e S$ as $\varphi_{1}(x)=e x$. Since $\varphi_{1}$ is a surjective morphism, $e S$ is connected. Define $\varphi_{2}: S \rightarrow e S f$ as $\varphi_{2}(x)=e x f$. Since $\varphi_{2}$ is a surjective morphism, eSf is connected. $S \times S$ is connected. Define $\varphi_{3}: S \times S \rightarrow S e S$ as $\varphi_{3}(x, y)=x e y$. If $S e S$ is closed, then $\varphi_{3}(S \times S)=S e S$ is also connected.

THEOREM 2.3. Let $S$ be a connected algebraic semigroup. Then

(1) all maximal subgroups of $S$ are closed if and only if $S$ is a nil extension of a completely simple semigroup.

(2) all subgroups of $S$ are trivial if and only if $S$ is a nil extension of a rectangular band.

Proof. (2) follows trivially from (1). So we prove (1). First assume that all maximal subgroups of $S$ are closed. Let $e \in E(S)$. By Proposition 2.2, eSe is connected. By hypothesis $H_{e}$ is closed. By Corollary 1.2, eSe $\backslash H_{e}$ is also closed. Hence $e S e=H_{e}$. Thus $a \mid e$ for all $a \in S$. Hence $e \in T=$ kernel of $S$. Thus $E(S) \subseteq T$. By Corollary $1.11, T$ is completely simple and $S / T$ is nil. Conversely assume $S / T$ is nil where $T$ is the completely simple kernel of $S$. Then for $e \in E(S)=E(T), H_{e}=e S e$ is closed.

TheOREM 2.4. Let $S$ be a connected algebraic semigroup. Then the following conditions are equivalent.

(1) All subgroups of the top $\mathcal{S}$-indecomposable component of $S$ are abelian.

(2) All subgroups of $S$ are abelian.

(3) eSe is commutative for all $e \in E(S)$.

Proof. (1) $\Rightarrow(3)$. Let $T$ be the top $S$-indecomposable component of $S$. Then by Theorem 1.12, $P=S \backslash T$ is closed. Let $e \in E(T)$. Then $H_{e}$ is abelian. Let $S_{1}=e S e, P_{1}=S_{1} \backslash H_{e}$. Then $P_{1}$ is closed, $S_{1}$ is closed and connected. $S_{1}=P_{1} \cup$ $H_{e}$. Let $a \in H_{e}$. Then $H_{e} \subseteq C_{S_{1}}(a)$ and so $S_{1}=P_{1} \cup C_{S_{1}}(a)$. Hence $C_{S_{1}}(a)=S_{1}$. Thus $H_{e} \subseteq C\left(S_{1}\right)$ and $S_{1}=P_{1} \cup C\left(S_{1}\right)$. Hence $C\left(S_{1}\right)=S_{1}$ and $S_{1}$ is commutative. Let $a \in T$. By Corollary 1.4 there exists $n \in Z^{+}$such that $a^{n} \mathcal{H}$ e for some $e \in E(T)$. So $a^{n} S a^{n} \subseteq e S e$ is commutative. Let $T_{1}=\left\{a \mid a \in S, a^{n} S a^{n}\right.$ is commutative $\}$. Then $T_{1}$ is closed, $T \subseteq T_{1}$. Since $S=P \cup T_{1}, T_{1}=S$. Hence eSe is commutative for all $e \in E(S)$. That $(3) \Rightarrow(2) \Rightarrow(1)$ is obvious.

THEOREM 2.5. Let $S$ be a connected algebraic semigroup such that all subgroups of $S$ are abelian. Suppose further that for each $a \in S$, there exists $e \in E(S)$ such that $e a=a e=a$. Then $S$ is a semilattice of nil extensions of completely simple semigroups and the top $\mathcal{S}$-indecomposable component of $S$ is completely simple.

Proof. By Theorem 2.4, eSe is commutative for all $e \in E(S)$. Let $a \in S$. Then there exists $e \in E(S)$ such that $e a=a e=a$. Let $x, y \in S^{1}$. Then xay xay = $x(e a e)(e y x e)(e a e) y=x(e a e)^{2}(e y x e) y=x a^{2} y x e y$. Hence $a^{2} \mid(x a y)^{2}$. By a paper by the author [14, Theorem 2.13], $S$ is a semilattice of nil extensions of completely simple semigroups. Let $T$ be the top $\delta$-indecomposable component of $S$. Then $T$ is a nil extension of a completely simple semigroup. Let $T_{1}=$ kernel of $T$. Then 
$E(T) \subseteq T_{1}$. Let $a \in T$. Then there exists $e \in E(S)$ such that $e a=a$. Clearly $e \in E(T)$. Hence $a \in T_{1}$ and $T=T_{1}$ is completely simple.

A semigroup is regular if $a \in a S a$ for all $a \in S$.

THEOREM 2.6. Let $S$ be a regular, connected algebraic semigroup such that all subgroups of $S$ are abelian. Then $S$ is a finite subdirect product of semigroups, each of which is either completely simple or completely 0-simple.

Proof. Let $e, f, g \in E(S)$ such that $e \geqslant f, e \geqslant g, f g g$. We claim that $f=g$. There exist $x, y \in S^{1}$ such that $x f y=g$. By Theorem 2.4 , eSe is commutative. So $g=$ exefeye $=$ efeeyeexe $=$ eyeexeefe. Hence $g=$ fyexe $=$ eyexf. Hence $g f=f g=$ $g$. So $f \geqslant g$. Similarly $g \geqslant f$ and $f=g$. Thus

$$
\begin{aligned}
& \text { for all } e, f, g \in E(S), \text { the conditions } \\
& e \geqslant f, e \geqslant g, f g g \text { together imply } f=g .
\end{aligned}
$$

By Munn [11], the principal factors of $S$ are completely simple or completely 0 -simple. Hence by (2) and Lallement [10, Theorem 2.17], $S$ is a subdirect product of completely simple and completely 0-simple semigroups. Since, by Theorem 1.7, $S$ has only finitely many $G$-classes, a close examination of [10] shows that the subdirect product can be chosen to be finite.

THEOREM 2.7. Let $S$ be a connected algebraic semigroup, $e \in E(S), e \notin C(S)$. Then $e \in B$ where $B$ is either an infinite, closed right zero subsemigroup of $S$ or an infinite, closed left zero subsemigroup of $S$.

Proof. By symmetry assume $\operatorname{dim} e S \geqslant \operatorname{dim} S e$. Define $\phi: e S \rightarrow e S e$ as $\varphi(a)=$ $a e$. Then $\varphi$ is a surjective morphism, $e S, S e, e S e$ are closed and connected. By [17, Chapter I, §6, Theorem 7], $\operatorname{dim} e S \geqslant \operatorname{dim} e S e$. First assume $\operatorname{dim} e S=\operatorname{dim} e S e$. Then since $e S e \subseteq e S, e S=e S e$. Since $\operatorname{dim} e S \geqslant \operatorname{dim} S e$ and $e S e \subseteq S e$ we have $S e=e S e$. Hence $e S=S e$ and $e \in C(S)$, a contradiction. So $\operatorname{dim} e S>\operatorname{dim} e S e$. Let $B=\varphi^{-1}(e)$. By [17, p. 60] $\operatorname{dim} B>0$. Hence $B$ is infinite. Let $a \in B$. Then $a \in e S$, $a e=e$. Hence $a=e a$. Let $a, b \in B$. Then $a b=a e b=e b=b$. This proves the theorem.

THEOREM 2.8. Let $S$ be a connected algebraic semigroup. Then the following conditions are equivalent.

(1) $E(S)$ is finite.

(2) $E(S)$ is commutative.

(3) $E(S) \subseteq C(S)$.

(4) $S$ is a semilattice of nil extensions of groups.

Proof. By Theorem 2.7, (1) $\Rightarrow(3)$ and (2) $\Leftrightarrow(3)$. By Corollary $1.8,(4) \Rightarrow(1)$. So it suffices to show that (3) $\Rightarrow$ (4). Assume (3). Let $a, b \in S$ such that $a b, b a \in E(S)$ $\subseteq C(S)$. Then $a b=a(b a) b=(a b)(b a)=b a b a=b a$. By Weissglass and the author [16, Corollary 8], we are done.

The following result is implicit in Munn [11]. We include a proof here for the convenience of the reader. 
LEMMA 2.9 [MUNN]. Let $S$ be a strongly $\pi$-regular semigroup. Let $a, b \in S$. If $a g a b$ then $a \mathcal{R} a b$. If $a \mathcal{G} b a$, then $a \mathfrak{L} b a$. If $a g a^{2}$, then $a \mathcal{H} a^{2}$.

Proof. It suffices to consider the case $a g a b$. There exist $x, y \in S^{1}$ such that $x a b y=a$. Then $x^{t} a(b y)^{t}=a$ for all $t \in \mathbf{Z}^{+}$. There exist $n \in \mathbf{Z}^{+}, e \in E(S)$ such that $(b y)^{n} \mathcal{H} e$. So $a=a e \in a(b y)^{n} S^{1} \subseteq a b S^{1}$. Hence $a \mathfrak{R} a b$.

LEMMA 2.10. Let $S$ be a connected algebraic semigroup, e, $f \in E(S)$, e|f. Then there exists $g \in E(S)$ such that $e^{\mathcal{R} g}$ and $g f £ f$.

Proof. Let $E=E(S)$. Suppose the lemma is false. Then by Lemma 2.9, $g f \nmid f$ for all $g \in E$ with $g \Re e$. In particular ef $\nmid f$. There exist $x, y \in S$ such that $x e y=f$. By Corollary 1.2 and Proposition 2.2, eSe $\backslash H_{e}$ and $f S f \backslash H_{f}$ are closed sets. Let

$$
T_{1}=\left\{a \mid a \in e S, \text { fxaf } \in f S f \backslash H_{f}\right\}, \quad T_{2}=\left\{a \mid a \in e S, a e \in e S e \backslash H_{e}\right\} .
$$

Then $T_{1}, T_{2}$ are closed subsets of $e S$. If $e \notin T_{1}$, then $f x e f \in H_{f}$ and $e f \mid f$, a contradiction. So $e \in T_{1}$. Clearly $f x e y f=f$ and so $e y \notin T_{1}$. Thus $\varnothing \neq T_{1} \varsubsetneqq e S$. Clearly $e \notin T_{2}$. We claim that ef $\in T_{2}$. Otherwise efe $\in H_{e}$. Then ef $\mid$ efe $|e| f$, a contradiction. So ef $\in T_{2}$. Hence $\varnothing \neq T_{2} \varsubsetneqq e S$. Since $e S$ is connected by Proposition 2.2, $T_{1} \cup T_{2} \neq e S$. Hence there exists $a \in e S$ such that $a \notin T_{1} \cup T_{2}$. Then $e a=a, f x a f \in H_{f}$, ae $\in H_{e}$. There exists $z \in S$ such that $z a e=e$. So $z a^{2}=z a e a$ $=e a=a$. Hence $a^{2} g a$. By Lemma 2.9, $a^{2} \mathcal{F}$ a. By [6, Theorem 2.16], there exists $g \in E$ such that $a \mathcal{H} g$. Now $g \in a^{2} S=a e a S \subseteq a e S=e S, e \in a e S \subseteq a S=g S$. So $e \Re g$. Now $f x a g f=f x a f \in H_{f}$. Hence $g f \mid f$, a contradiction. This proves the lemma.

THEOREM 2.11. Let $S$ be a connected algebraic semigroup such that $E(S)$ is a subsemigroup of $S$. Then $S$ is a semilattice of nil extensions of rectangular groups.

Proof. Let $E=E(S)$. Let $a, b \in S$ such that $e=a b, f=b a \in E$. By the author [14, Theorem 2.17], it suffices to show that $f e f=f$. Now $e=a b \mid(b a)^{2}=f$. By Lemma 2.10 there exists $g \in E$ such that $e \Re g, g f \varrho f$. Since $g f \in E, f g f=f$. Since $e \Re g$, eg $=g$. So $f e g f=f$. Since $f e \in E, f e f=(f e)^{2} g f=f e g f=f$. This proves the theorem.

THEOREM 2.12. Let $S$ be a connected algebraic semigroup such that all subgroups of $S$ are abelian. Then the following conditions are equivalent.

(1) $E(S)$ is a band.

(2) $E(S)$ is a normal band.

(3) $S$ is a semilattice of nil extensions of rectangular groups.

Proof. (1) $\Rightarrow$ (3) follows from Theorem 2.11. (2) $\Rightarrow(1)$ is obvious. So we must show (3) $\Rightarrow(2)$. By Corollary 1.4, there exists $n \in \mathbf{Z}^{+}$such that for all $a \in S, a^{n}$ ICe for some $e \in E(S)$. Let $E=E(S)$ and let $T$ be the top $\mathcal{S}$-indecomposable component of $S$. If $T=S$, we are done. So assume $P=S \backslash T \neq \varnothing$. $P$ is a prime ideal of $S$ and hence closed by Theorem 1.12. $T$ is a nil extension of a rectangular 
group $T_{1}$. Since the subgroups of $T_{1}$ are abelian, $T_{1}$ satisfies the identity $x y z w=$ $x z y w$. Hence $T$ satisfies the identity $x^{n} y^{n} z^{n} w^{n}=x^{n} z^{n} y^{n} w^{n}$. By [17, p. 54], $S \times S \times$ $S \times S$ is connected. Let $M=\left\{(a, b, c, d) \mid a, b, c, d \in S, a^{n} b^{n} c^{n} d^{n}=a^{n} c^{n} b^{n} d^{n}\right\}$. Then $M$ is closed and $T \times T \times T \times T \times \subseteq M$. Clearly

$$
\begin{aligned}
S \times S \times S \times S= & M \cup(S \times S \times S \times P) \cup(S \times S \times P \times S) \\
& \cup(S \times P \times S \times S) \cup(P \times S \times S \times S) .
\end{aligned}
$$

Hence $M=S \times S \times S \times S$. Thus for all $e, f, g, h \in E(S)$, efgh $=e g f h$. In particular efef $=$ eeff $=e f$ and $E(S)$ is a normal band.

THEOREM 2.13. Let $S$ be a connected algebraic semigroup such that $\operatorname{dim} S=1$. Then $S$ is either a group, a group with zero, a null semigroup, a right zero semigroup or a left zero semigroup.

Proof. First assume $S$ has an identity element 1 . If $E(S)=\{1\}$, then $S$ is a group. Otherwise there exists $e \in E(S)$ such that $e \neq 1$. Then $\operatorname{dim} e S=\operatorname{dim} S e=$ 0 . So $e S=S e=\{e\}$. Hence $S$ has a zero 0 and $E(S)=\{1,0\}$. Let $G$ be the group of units of $S$. By Corollary 1.2, $M=S \backslash G$ is a closed ideal of $S$. Let $a \in M$. Consider the map $\varphi: S \rightarrow M$ given by $\varphi(x)=a x . \varphi$ is a morphism. Hence $T$, the closure of $\varphi(S)$ is irreducible. Since $T \subseteq M \neq S, \operatorname{dim} T=0$. Since $0, a \in T$, $a=0$. Thus $S=G \cup\{0\}$.

So assume $S$ does not have an identity element. Let $e \in E(S)$. Suppose $e S=S$. Then $S e \neq S$. So $S e=\{e\}$. Let $a, b \in S$. Then $a b=a(e b)=(a e) b=e b=b$. So $S$ is a right zero semigroup. Similarly $S e=S$ implies that $S$ is a left zero semigroup. So assume $e S \neq S, S e \neq S$ for all $e \in E(S)$. Hence $e S=S e=\{e\}$. So $S$ has a zero 0 and $E(S)=\{0\}$. By Corollary 1.4, there exists $n \in \mathbf{Z}^{+}$such that $a^{n}=0$ for all $a \in S$. Let $D=\left\{a \mid a \in S, a^{2}=0\right\}$. Then $D$ is closed. Define $\varphi$ : $S \rightarrow D$ as $\varphi(a)=a^{n-1}$. Then $\varphi(S) \neq\{0\}$. Let $T$ be the closure of $\varphi(S)$. Then $T \subseteq D$. Since $S$ is connected, $T$ is irreducible. So $\operatorname{dim} T=1$ and $S=T=D$. Let $a \in S$. Let $M=\{b \mid b \in S, a b=0\}$. We claim that $M=S$. Suppose not. Clearly $M$ is closed. Define $\psi: S \rightarrow M$ as $\psi(b)=a b$. Since $M \neq S, \psi(S) \neq\{0\}$. If $W$ is the closure of $\psi(S)$, then $\operatorname{dim} W \neq 0, W$ is irreducible, $W \subseteq M$. This contradiction shows that $M=S$. Hence $S^{2}=\{0\}$, proving the theorem.

REMARK 2.14. It is well known [2, p. 257] that a connected algebraic group of dimension one is *-isomorphic to either $(K,+)$ or the group $\{(a, b) \mid a, b \in K$, $a b=1\}$ under multiplication. Let $S$ be an algebraic semigroup of dimension 1 . The only case of Theorem 2.13 that needs a closer look is when $S=G^{0}, G$ is a group. Let 1 be the identity of $S$. Then $\hat{G}=\{(a, b) \mid a, b \in S, a b=1\}$ is a connected algebraic group of dimension 1 . So $S$ is isomorphic to $(K, \cdot)$. The example $S=\left\{(x, y) \mid x, y \in K, x^{2}=y^{3}\right\}$ under multiplicaton shows that in general $S$ is not *-isomorphic to $(K, \cdot)$.

THEOREM 2.15. Let $S$ be a connected algebraic semigroup such that $\operatorname{dim} S=2$. Then $E(S)$ is a band. If $S$ does not have an identity element then $E(S)$ is a normal band. 
Proof. Let $M$ be the kernel of $S$. By Corollary $1.5, M$ is closed and completely simple. Let $e \in E(M)$. Then $S e S=M$. By Proposition 2.2, $M$ is connected. First assume $M=S$. If $e S=S$ for some $e \in E(S)$, it follows (since $S$ is completely simple) that $E(S)$ is a right zero semigroup. Similarly $S e=S$ implies $E(S)$ is a left zero semigroup. So assume $e S \neq S, S e \neq S$ for all $e \in E(S)$. So $\operatorname{dim} e S=\operatorname{dim} S e$ $\leqslant 1$ for all $e \in E(S)$. Let $e \in E(S)$. If $e S e \neq\{e\}$ then $\operatorname{dim} e S e=1$. Since $e S e \subseteq$ $S e \cap e S$, we obtain $e S=S e$. But then $S$ is a group. So assume $e S e=\{e\}$ for all $e \in E(S)$. Then $S$ is a rectangular band.

Next assume $\operatorname{dim} M=1$. By Theorem $2.13, M$ is either a right zero semigroup, a left zero semigroup or a group. By symmetry assume $M$ is not a left zero semigroup. If $E(M)=E(S)$, we are done. So assume $E(M) \neq E(S)$. Suppose $S$ has an identity element 1 . Let $e \in E(S), e \notin M$. Then $M \varsubsetneqq e S$. So $e S=S$ and $e=1$. Then $E(S)=E(M) \cup\{1\}$ and we are done. Next assume $S$ does not have an identity element. Let $e \in E(S) \backslash M$. As above, $e S=\mathrm{S}$. So $S e \neq S$. Now $M e$ is closed and connected and $M e \varsubsetneqq S e$. So $\operatorname{dim} M e=0$. If $M e=\{f\}$, let $\theta(e)=f \in$ $E(M)$. So $\theta: E(S) \backslash M \rightarrow E(M)$. Let $D_{1}=E(M), D_{2}=E(S) \backslash D_{1}$. Then $D_{1}, D_{2}$ are right zero semigroups. If $e \in D_{2}, f \in D_{1}$, then $e f=f, f e=\theta(e)$. It follows easily that $E(S)$ is a normal band.

Finally assume that $\operatorname{dim} M=0$. Then $S$ has a zero 0 . Suppose $S$ has an identity element 1. Let $e \in E(S), e \neq 1,0$. Then $\{0\} \varsubsetneqq e S e \subseteq e S \varsubsetneqq S$. So $e S=e S e$. Similarly $S e=e S e$ and $e \in C(S)$. Hence $E(S) \subseteq C(S)$. Next assume $S$ does not have an identity element. By symmetry we can assume that $e S \neq S$ for all $e \in E(S)$. Then $\{0\} \varsubsetneqq e S e \subseteq e S \varsubsetneqq S$ for all $e \in E(S), e \neq 0$. So $e S e=e S$ for all $e \in E(S), e \neq 0$. Let $A=\{e \mid e \in E(S), S e=s\}$. Then $A=\varnothing$ or $A$ is a left zero semigroup. Let $e \in E(S), e \neq 0, e \notin A$. Then $\{0\} \varsubsetneqq e S e \subseteq S e \varsubsetneqq S$. Hence $e S e=S e$ and $e S=S e$. So $e \in C(S)$. It follows that $E(S)$ is a normal band.

Let $S$ be a strongly $\pi$-regular semigroup, $J$ a regular $G$-class of $S$. Let $J^{0}$ be the semigroup $J \cup\{0\}$ where 0 is the zero of $J^{0}$ and for $a, b \in J$, we set $a b=0$ if $a b \notin J$. By Munn [11], $J^{0}$ is completely 0-simple. By the Rees theorem [6, Theorem 3.5] we can assume that $J^{0}=(\Gamma \times G \times \Lambda) \cup\{0\}$ with sandwich map $P: \Lambda \times \Gamma \rightarrow$ $G^{0}$ where $G$ is a group. Multiplication in $J^{0}$ is given by

$$
(\alpha, a, \beta)(\gamma, b, \delta)= \begin{cases}(\alpha, a P(\beta, \gamma) b, \delta) & \text { if } P(\beta, \gamma) \neq 0 \\ 0 & \text { if } P(\beta, \gamma)=0\end{cases}
$$

THEOREM 2.16. Let $S$ be a connected algebraic semigroup, $J$ a regular $\mathcal{G}$-class of $S$. Let $J^{0}$ have the Rees representation given by (3). Then for all $\alpha, \beta \in \Gamma$, there exists $\gamma \in \Lambda$ such that $P(\gamma, \alpha) \neq 0$ and $P(\gamma, \beta) \neq 0$. For all $\gamma, \delta \in \Lambda$, there exists $\alpha \in \Gamma$ such that $P(\gamma, \alpha) \neq 0$ and $P(\delta, \alpha) \neq 0$.

Proof. The second statement being the dual of the first, we only need to prove the first. Let $\alpha, \beta \in \Gamma$. Since $J^{0}$ is regular, it follows [6, Lemma 3.1] that there exist $\mu, \nu \in \Lambda$ such that $P(\mu, \alpha) \neq 0, P(\nu, \beta) \neq 0$. Let $e=\left(\alpha, P(\mu, \alpha)^{-1}, \mu\right), f=$ $\left(\beta, P(\nu, \beta)^{-1}, \nu\right)$. Then $e, f \in E(S), e \mid f$. By Lemma 2.10, there exists $g \in E(S)$

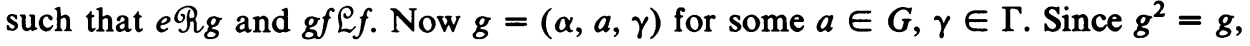
$P(\gamma, \alpha) \neq 0$. Since $g f \neq 0$ in $J^{0}, P(\gamma, \beta) \neq 0$. This proves the theorem. 
Theorem 2.17. Suppose $S$ is a connected, algebraic semigroup. Assume that $S$ is a semilattice of groups and that $E(S)$ is linearly ordered. Then $|E(S)|<2$.

Proof. By Theorem 2.8, $E(S)$ is finite and $E(S) \subseteq C(S)$. Suppose $|E(S)| \geqslant 3$. Let $E(S)=\left\{e_{1}<e_{2}<e_{3}<\cdots\right\}$. Let $T=e_{3} S$. Let $T_{1}=e_{1} S, T_{2}=e_{2} S$. Then $T_{1} \varsubsetneqq T_{2} \varsubsetneqq T, e_{2}\left(T \backslash T_{1}\right)=T_{2} \backslash T_{1}, e_{2} T_{1}=T_{1}$. Define $\varphi: T \rightarrow T_{2}$, as $\varphi(x)=e_{2} x$. Clearly $\varphi$ is surjective and $\operatorname{dim} T>\operatorname{dim} T_{2}$. So [17, p. 60], $\operatorname{dim} \varphi^{-1}(a)>0$ for all $a \in T_{2}$. In particular $\operatorname{dim} \varphi^{-1}\left(e_{1}\right)>0$. Let $x \in \varphi^{-1}\left(e_{1}\right)$. Then $e_{2} x=e_{1}$. But then $x \in T_{1}$ and so $e_{2} x=x$. This contradiction proves the theorem.

3. Examples and problems. Let $D$ be a closed subset of $K^{n}$. Let $\circ$ be a binary operation on $D$ such that the map $(a, b) \rightarrow a \circ b$ from $D \times D$ into $D$ is a morphism. We will then say that $(D, \circ)$ is an algebraic groupoid.

EXAMPLE 3.1. Let $D$ be an algebraic groupoid, $S$ a subsemigroup of $D$. Let $T$ be the closure of $S$ in $D$. Then $T$ is an algebraic semigroup. In fact let $a \in S$, $T_{1}=\{b \mid b \in T, a b \in T\}$. Then $S \subseteq T_{1}$ and so $T_{1}=T$. So a $T \subseteq T$. Let $T_{2}=$ $\{b \mid b \in T, b T \subseteq T\} . S \subseteq T_{2}$ and so $T_{2}=T$. Hence $T^{2} \subseteq T$. Let $a, b \in S$ and let $T_{3}=\{c \mid c \in T,(a b) c=a(b c)\} . S \subseteq T_{3}$ and so $T_{3}=T$. Repeating this argument twice, we see that $T$ is a semigroup.

EXample 3.2. Let $X \subseteq K^{n}$ be closed. Let $S=\left\{A \mid A \in \Re_{n}(K), X A \subseteq X\right\}$. Then $S$ is a closed subsemigroup of $\mathfrak{T}_{n}(K)$.

EXAMPLe 3.3. Let $X \subseteq K^{n}$ be a nonempty closed set. Then $X$ admits a right zero, left zero and null semigroup structures given by $a b=b, a b=a, a b=u$ where $u$ is a fixed element of $X$.

EXAMPLE 3.4. Let $S$ be any finite semigroup. Then $S$ is closed subsemigroup of the finite dimensional algebra $K[S]$. Hence $S$ is an algebraic semigroup.

EXAMPLE 3.5. Let $S \subseteq K^{2}$ be the closed set $\left\{(a, b) \mid a, b \in K, a b^{2}=b\right\}$. If $(a, b)$, $(c, d) \in S$, define $(a, b)(c, d)=(a b c d a c+1-a b c d, 0)$. Then $S$ is a commutative algebraic semigroup. Note that $(1,1) S=S^{2}=\{(a, 0) \mid a \in K, a \neq 0\}$ is not closed. $S^{3}=\{(1,0)\}$.

Problem 3.6. Let $S$ be an algebraic semigroup. Does there exist $n \in \mathbf{Z}^{+}$such that $S^{n}=S^{n+1}$ is closed?

Problem 3.7. Let $S$ be an algebraic semigroup, $e \in E(S)$. Is $S e S$ necessarily closed?

Problem 3.8. Can the ideals in Corollary 1.11 be chosen to be closed?

Problem 3.9. Let $n \in \mathbf{Z}^{+}$. Does the number of regular $g$-classes of strongly $\pi$-regular subsemigroups of $\mathfrak{N}_{n}(K)$ have an upper bound (depending on $n$ )? More generally, can $\mathcal{E}$ in Lemma 1.6 be replaced by a sufficiently large finite set of idempotents?

Problem 3.10. Are the nil Rees factor semigroups of Theorem 1.10 and Corollary 1.11 necessarily nilpotent?

Problem 3.11. Can the Krohn-Rhodes theorem for finite semigroups be generalized to strongly $\pi$-regular subsemigroups of $\Re_{n}(K)$ ?

EXAMPLE 3.12. Let $T_{1} \subseteq K^{m}, T_{2} \subseteq K^{n}$ be algebraic semigroups. Let $S=\left(T_{1} \times\right.$ $\left.\left\{0_{n}\right\} \times\{1\}\right) \cup\left(\left\{0_{m}\right\} \times T_{2} \times\{0\}\right) \subseteq K^{m+n+1}$ where $0_{m}, 0_{n}$ are the zero vectors of 
$K^{m}$ and $K^{n}$ respectively. Then $S$ is closed. Define multiplication in $S$ as follows. $(a, b, \alpha)(c, d, \beta)=(\alpha \beta a c,(1-\alpha) \beta b+\alpha(1-\beta) d+(1-\alpha)(1-\beta) b d, \alpha \beta)$. Then $S$ is an algebraic semigroup. Let $\hat{T}_{1}=T_{1} \times\left\{0_{n}\right\} \times\{1\}, \hat{T}_{2}=\left\{0_{m}\right\} \times T_{2} \times$ $\{0\}$. Then $S=\hat{T}_{1} \cup \hat{T}_{2}, x y=y x=y$ for $x \in \hat{T}_{1}, y \in \hat{T}_{2} . \hat{T}_{1}, \hat{T}_{2}$ are disjoint closed subsemigroups of $S . \hat{T}_{i}$ is ${ }^{*}$-isomorphic to $T_{i}(i=1,2)$.

EXAMPLE 3.13. Let $\mathcal{Q}$ be a finite dimensional algebra over $K$. Then the multiplicative semigroup of $Q$ is a connected algebraic semigroup. $Q$ along with the circle operation $a \circ b=a+b-a b$ is also a connected algebraic semigroup.

EXAMPLE 3.14. Let $S=\Re_{n}(K)$. For $i=1, \ldots, n$, let $S_{i}=\{a \mid a \in S, \rho(a)<i\}$. If $e \in S_{i}, e^{2}=e, \rho(e)=i$, then $S e S=S_{i}$ and so by Proposition 2.2, each $S_{i}$ is a connected algebraic semigroup. $S_{1}$ is completely 0 -simple and all subgroups of $S_{1}$ are abelian. Also $\operatorname{dim} S_{1}=2 n-1$.

Let $S, T$ be algebraic semigroups. Suppose for $a \in S, b \in T$ an element $a^{b} \in S$ is uniquely determined. Suppose the map $(a, b) \rightarrow a^{b}$ is a morphism and that for all $a_{1}, a_{2} \in S, b_{1}, b_{2} \in T,\left(a_{1} a_{2}\right)^{b}=a_{1}^{b} a_{2}^{b},\left(a_{1}\right)^{b_{1} b_{2}}=\left(a_{1}^{b_{2}}\right)^{b_{1}}$. In $D=S \times T$ define $\left(a_{1}, b_{1}\right)\left(a_{2}, b_{2}\right)=\left(a_{1} a_{2}^{b_{1}}, b_{1} b_{2}\right)$. Then the semidirect product $D$ is an algebraic semigroup. If $S, T$ are connected then so is $D$. In particular if $a \in \mathfrak{T}_{n}(K), b \in$ $G L(n, K)=\left\{a \mid a \in \Re_{n}(K), \rho(a)=n\right\}$, we can set $a^{b}=b a b^{-1}$. If $G$ is any connected, closed subgroup of $G L(n, K)$, we can form the semidirect product of $S_{i}$ (see Example 3.14) and $G$ to again obtain a connected algebraic semigroup. By Lallement [10, Theorem 2.17], the semidirect product of $S_{1}$ and $G$ is a subdirect product of completely simple and completely 0 -simple semigroups.

EXAMPLe 3.15. The example $S=\left\{\left(\begin{array}{ll}1 & a \\ 0 & b\end{array}\right) \mid a, b \in K\right\}$ shows that Theorem 2.12 is not true without the assumption that the subgroups of $S$ are abelian.

Problem 3.16. Let $S$ be a connected algebraic semigroup which is a semilattice of groups. Determine all possibilities for $E(S)$ and $|E(S)|$. For example, by Theorems 1.2 and $2.17,|E(S)| \neq 3$. If $S$ is also the multiplicative semigroup of a finite-dimensional algebra, then clearly $|E(S)|=2^{n}$ for some $n \in \mathbf{Z}^{+}$. This is not true in general as the following example shows.

EXAMPLE 3.17. Let $T=K^{4}$ under multiplicaton and let $S=$ $\{(a, b, c, d) \mid a, b, c, d \in K, a b=c d\}$. Then $S$ is a connected, closed subsemigroup of $T . S$ is also a semilattice of groups, $\operatorname{dim} S=3$ and $|E(S)|=10$.

Problem 3.18. Determine all possibilities for $\Omega(S)$ and $|\Omega(S)|$ where $S$ is a connected algebraic semigroup.

EXAMPLE 3.19. Let $T_{1}=\left(K^{3}, *\right)$ where

$$
\left(a_{1}, a_{2}, a_{3}\right) *\left(b_{1}, b_{2}, b_{3}\right)=\left(a_{2} b_{3}+a_{1}+b_{1}, b_{2}, a_{3}\right) \text {. }
$$

Let $T_{2}$ be any commutative finite-dimensional algebra with an identity element. Then $T_{1}$ is completely simple. $T_{1}, T_{2}, T_{1} \times T_{2}$ are all examples of connected algebraic semigroups satisfying the hypothesis of Theorem 2.5 .

EXAMPLE 3.20. Let $S=\left\{\left(\begin{array}{ll}a & b \\ 0 & 0\end{array}\right) \mid a, b \in K\right\}$. Then $S$ is a connected algebraic semigroup of dimension $2 . S$ is a semilattice of a nil semigroup and a right group.

EXAMPLE 3.21. Let $P \in \mathfrak{R}_{n}(K)$ and let $\mathbb{Q}=\left\{A \mid A \in \mathfrak{R}_{n}(K), A^{T} P A=0\right\}$, $\mathscr{B}=\left\{A \mid A \in \mathfrak{T}_{n}(K), A^{T} P A=P\right\}$. Then $\mathscr{Q}, \mathscr{B}$ are closed subsemigroups of 
$\mathfrak{K}_{n}(K) . \mathscr{Q}$ has a zero and $\mathscr{B}$ has an identity element. When is $\mathscr{Q}$ or $\mathscr{B}$ connected? If $n=3$ and

$$
P=\left(\begin{array}{ccc}
0 & 1 & 0 \\
-1 & 0 & 0 \\
0 & 0 & 0
\end{array}\right),
$$

then $Q$ is a connected algebraic semigroup of dimension 7 .

\section{REFERENCES}

1. G. Azumaya, Strongly $\pi$-regular rings, J. Fac. Sci. Hokkaido Univ. 13 (1954), 34-39.

2. A. Borel, Linear algebraic groups, Benjamin, New York, 1969.

3. W. E. Clark, Remarks on the kernel of a matrix semigroup, Czechoslovak Math. J. 15 (90) (1965), 305-310.

4. __ Affine semigroups over an arbitrary field, Proc. Glasgow Math. Assoc. 7 (1965), 80-92.

5. Private communication.

6. A. H. Clifford and G. B. Preston, The algebraic theory of semigroups, Vol. 1, Math. Surveys, No. 7, Amer. Math. Soc., Providence, R. I., 1961.

7. P. M. Cohn, Universal algebra, Harper and Row, New York, 1965.

8. M. Demazure and P. Gabriel, Groupes algébriques. Tome I: Géométrie algébrique, généralités, groups commutatifs, North-Holland, Amsterdam, 1970.

9. M. P. Drazin, Pseudo-inverses in associative rings and semigroups, Amer. Math. Monthly 7 (1958), 506-514.

10. G. Lallement, Demi-groupes réguliers, Ann. Mat. Pura Appl. 77 (1967), 47-129.

11. W. D. Munn, Pseudo-inverses in semigroups, Proc. Cambridge Philos. Soc. 57 (1961), 247-250.

12. M. Petrich, Associative polynomial multiplications over an integral domain, Math. Nachr. 29 (1965), 67-75.

13. R. J. Plemmons and R. Yoshida, Generating polynomials for finite semigroups, Math. Nachr. 47 (1970), 69-75.

14. M. S. Putcha, Semilattice decompositions of semigroups, Semigroup Forum 6 (1973), 12-34.

15. __ Quadratic semigroups on affine spaces, Linear Algebra and Appl. 26 (1979), 107-121.

16. M. S. Putcha and J. Weissglass, A semilattice decomposition into semigroups with at most one idempotent, Pacific J. Math. 39 (1971), 225-228.

17. I. R. Shafarevich, Basic algebraic geometry, Springer-Verlag, Berlin and New York, 1974.

18. T. Tamura, The theory of construction of finite semigroups. I, Osaka J. Math. 8 (1956), 243-261.

19. __ Another proof of a theorem concerning the greatest semilattice decomposition of a semigroup, Proc. Japan Acad. 40 (1964), 777-780.

20. T. Tamura and N. Kimura, On decomposition of a commutative semigroup, Kōdai Math. Sem. Rep. 4 (1954), 109-112.

21. R. Yoshida, Algebraic systems which admit polynomial operations, Mem. Res. Inst. Sci. Engrg. Ritsumeikan Univ. 10 (1963), 1-5.

22. , On semigroups, Bull. Amer. Math. Soc. 69 (1963), 369-371.

Department of Mathematics, North Carolna State University, Raleigh, North Carolina 27650 